\title{
X-ray Detection of Pulsar PSR B1757-24 and its Nebular Tail
}

\author{
V. M. Kaspi, ${ }^{1,2,3}$ E. V. Gotthelf, ${ }^{4}$ B. M. Gaensler, ${ }^{2,5}$ M. Lyutikov ${ }^{1,6}$
}

\begin{abstract}
We report the first X-ray detection of the radio pulsar PSR B1757-24 using the Chandra X-ray Observatory. We detect point-source emission at the pulsar position plus a faint tail extending nearly $20^{\prime \prime}$ east of the pulsar, in the same direction and with comparable morphology to the radio tail. Assuming the point-source X-ray emission is magnetospheric, the observed X-ray tail represents only $\sim 0.01 \%$ of the pulsar's spindown luminosity. This is significantly lower than the analogous efficiencies of most known X-ray nebulae surrounding rotation-powered pulsars. Assuming a non-thermal spectrum for the tail photons, we show that the tail is unlikely to be emission left behind following the passage of the pulsar, but rather is probably from synchrotronemitting pulsar wind particles having flow velocity $\sim 7000 \mathrm{~km} \mathrm{~s}^{-1}$. We also show that there must be a significant break in the tail synchrotron spectrum between the radio and X-ray bands that is intrinsic to the particle spectrum. No emission is detected from the shell supernova remnant G5.4-1.2. The upper limits on remnant emission are unconstraining.
\end{abstract}

Subject headings: pulsars: general — pulsars: individual (PSR B1757-24) — ISM: individual (G5.4-1.2) — X-rays: general

\section{Introduction}

PSR B1757-24 is a 124-ms radio pulsar discovered near the supernova remnant G5.4-1.2 (Manchester et al. 1985). Radio timing observations established that the pulsar has characteristic age $16 \mathrm{kyr}$ and spin-down luminosity $\dot{E}=2.6 \times 10^{36} \mathrm{erg} \mathrm{s}^{-1}$ (Manchester et al. 1991). The pulsar is

\footnotetext{
${ }^{1}$ Department of Physics, Rutherford Physics Building, McGill University, 3600 University Street, Montreal, Quebec, H3A 2T8, Canada

${ }^{2}$ Department of Physics and Center for Space Research, Massachusetts Institute of Technology, Cambridge, MA 02139

${ }^{3}$ Canada Research Chair, Alfred P. Sloan Fellow

${ }^{4}$ Columbia University Astronomy Department, Pupin Hall, 550 West 120th Street, New York, NY 10027

${ }^{5}$ Hubble Fellow; current address Harvard-Smithsonian Center for Astrophysics, 60 Garden Street, Cambridge, MA 02138

${ }^{6}$ CITA National Fellow
} 
at the tip of a flat-spectrum radio protuberance just outside the west side of the supernova remnant (SNR) shell. The protuberance consists of a small, roughly circular nebula, G5.27-0.90, from which a collimated jet-like feature is emerging on its western side. The pulsar is at the westernmost tip of the collimated feature. The morphology and spectrum of the jet are strongly suggestive of a ram-pressure confined pulsar wind nebula (PWN) (Frail \& Kulkarni 1991). Thus, assuming an association between the pulsar and G5.4-1.2, the former appears to have overtaken the expanding shell, presumably because of a large kick at birth. Given the angular displacement of the pulsar from the best-estimate remnant center, and if the pulsar's characteristic age is a good estimate of

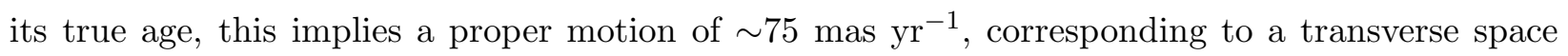
velocity of $v_{t} \sim 1800 \mathrm{~km} \mathrm{~s}^{-1}$ (Frail et al. 1994), for a distance of $5 \mathrm{kpc}$ (Caswell et al. 1987; Frail et al. 1994). However, recent interferometric observations have failed to detect the implied proper motion (Gaensler \& Frail 2000). They set a $5 \sigma$ upper limit on the proper motion of $<25 \mathrm{mas} \mathrm{yr}^{-1}$, corresponding to $v_{t}<590 \mathrm{~km} \mathrm{~s}^{-1}$. This suggests that the pulsar is older than its characteristic age, or that the assumed pulsar birth place is incorrect.

A ram-pressure confined pulsar wind should radiate X-rays as part of the broad-band synchrotron spectrum that results from the shock-acceleration and subsequent gyration of relativistic wind electron/positron pairs in the ambient magnetic field. We report here on Chandra X-ray Observatory observations of PSR B1757-24 in which we detect the source for the first time in X-rays.

\section{Observations and Results}

The PSR B1757-24/G5.4-1.2 field was observed using the Advanced CCD Imaging Spectrometer (ACIS) instrument aboard the Chandra X-ray Observatory on 2000 April 12. The ACIS CCD camera (Burke et al. 1997) is sensitive to X-rays in the $0.2-10 \mathrm{keV}$ band with 0.492 pixels. The pulsar was positioned on the back-illuminated S3 chip of the ACIS-S array, offset from the aimpoint by -0.37 in the Y-direction, in order to avoid source counts from being split between two readout nodes. Data were collected in the nominal timing mode, with 3.241 s exposures between CCD readouts, and "FAINT" spectral mode. The standard Chandra screening criteria produced a total usable exposure time of $19.6 \mathrm{ks}$.

The ACIS image reveals a faint point source near the radio pulsar position, and a fainter tail of emission on the eastern side, similar to the radio emission (Figure 1). Figure 2 shows the distribution of counts as a function of distance from the point source (with the point source excluded) in two $30^{\circ}$ wedges on the eastern and western sides of the pulsar, along with the mean of the field. With respect to the mean and the western side, the eastern side clearly has an overdensity of counts extending nearly $20^{\prime \prime}$ from the point source. Simulations of a point source at the pulsar position using the Chandra simulator MARX are consistent with what is observed in all directions but east. As the tail is in the chip readout direction, we were concerned about it being an instrumental artifact. However, trails in ACIS images aligned with the readout direction are only seen for bright 
sources, are symmetric about them, and have uniform intensity along the readout axis (M. Bautz, D. Edgar, H. Marshall, personal communication). Furthermore, we verified that there were no pointing anomalies during the observation and that the count rate for the tail photons is uniform in time over the observation. We therefore conclude that the extended, collimated emission we have detected is genuinely astrophysical.

The standard processing for ACIS produces measured source coordinates with a nominal accuracy quoted as 0.6 , however several problems are known to exist with the plate scale solution at the time of writing ${ }^{7}$. We derived our own astrometric solution by comparing field stars in our observation with catalogued optical coordinates. The X-ray source positions were measured using the CIAO source detection software celldetect with the cleaned $0.3-10 \mathrm{keV}$ Level 2 event file as input. For a signal-to-noise threshold set to 3 and the background estimated using ldetect, we found 9 significant X-ray sources within $5^{\prime}$ of the pulsar position. The on-line USNO-A2.0 astrometric reference catalogs (Monet et al. 1996) provided likely counterparts for 8 of the 9 X-ray sources. The USNO catalog gives stellar positions to an nominal $1 \sigma$ error of $\approx 0$ '.25 (Deutsch 1999, Monet, personal communication) although proper motion has not been applied. The offsets between the optical and X-ray positions are well clustered, strongly suggesting correct identifications. The average offset is $\sim 00^{\prime \prime} 6$, consistent with the expected systematic error in the absolute coordinates of ACIS. The calibrated position of the putative X-ray counterpart to the pulsar is RA $18^{\mathrm{h}} 01^{\mathrm{m}} 00^{\mathrm{s}} .027, \mathrm{DEC}-24^{\circ} 51^{\prime} 27^{\prime \prime} .53$ (J2000) with RMS error $0^{\prime \prime} 31$. This is within $1.2 \sigma$ of the radio pulsar location found by Gaensler \& Frail (2000). Thus, the point source is consistent with being the X-ray counterpart of the pulsar.

To characterize the energy dependence of the emission from the point source, a spectrum was extracted from a circular region having radius 7 pixels (3."4). An annular background region was chosen (approximately centered on the point source) having inner and outer radii 33 and 98 pixels, respectively. We found a total of 438 source counts and an estimated 8 background counts in the source region. We note that the background on the S3 chip was dominated by scattered emission from the nearby bright X-ray binary GX 5-1. The CIAO tools mkrmf and mkarf were used to generate common response files for the source and background regions. The data were regrouped such that spectral bins contained a minimum of 15 counts, resulting in 27 independent spectral bins. We fit the spectrum with the spectral analysis package XSPEC (version 11). We characterize the spectrum using two different models: a power law and thermal bremsstrahlung, each photoelectrically absorbed. Both provide acceptable fits, although the plasma temperature in the thermal model is poorly constrained. The results are shown in Table 1 . We verified using XSPEC that the expected $\sim 2.6 \%$ pileup fraction does not have a significant impact on the fitted parameters.

The tail east of the pulsar is much fainter than the point source. We attempted to characterize its spectrum by assuming a power-law model and holding the equivalent neutral hydrogen column

\footnotetext{
${ }^{7}$ http://asc.harvard.edu/mta/ASPECT/aspect_caveats.html
} 
density $N_{H}$ fixed at the value determined from the point source (see Table 1 ). We extracted a spectrum for the tail using a box having dimensions $40 \times 18$ pixels, which contained 118 counts. We used the same annulus to estimate the background as we did for the point source; in this way we estimate that approximately 40 counts were from the background, leaving 78 source counts. Spectral fits to the tail in the $0.3-9 \mathrm{keV}$ range for a power-law model yielded a mediocre fit to the data $\left(\chi_{\nu}^{2}=2.0\right.$ for 4 degrees of freedom). The best-fit power-law photon index of $1.0 \pm 0.6$ is consistent with relatively hard emission, as implied by the existence of a significant number of counts above $5 \mathrm{keV}$. However, given the $90 \%$ confidence range for $N_{H}$ (Table 1), we found that photon indexes in the range -0.32 to 1.8 are consistent with the data. Lower values of $N_{H}$, corresponding to lower values of the photon index, give slightly better fits. Improved characterization of the spectrum of the tail must await a deeper observation of the source. Nevertheless, we can determine the absorved surface brightness in the $2-8 \mathrm{keV}$ band to be $4.5 \times 10^{-16} \mathrm{erg} \mathrm{s}^{-1} \mathrm{~cm}^{-2} \operatorname{arcsec}^{-2}$, with uncertainty of $\sim 30 \%$. The total unabsorbed flux in the $2-8 \mathrm{keV}$ band in our extraction region is $9.8 \times 10^{-14} \mathrm{erg} \mathrm{s}^{-1} \mathrm{~cm}^{-2}$, with similar uncertainty.

\section{Discussion}

\subsection{The Pulsar}

Given the spatial coincidence of the X-ray point source with the radio pulsar position, the former is likely to be emission from the radio pulsar itself, in particular, non-thermal pulse-phaseaveraged magnetospheric emission. The observed 2-10 keV flux (Table 1) implies an unabsorbed luminosity, for a distance $d=5 \mathrm{kpc}$, of $2 \times 10^{33} \mathrm{erg} \mathrm{s}^{-1}$, assuming beaming angle $\phi=\pi \mathrm{sr}$. This implies an efficiency of conversion of spin-down luminosity into magnetospheric emission of $0.00020(\phi / \pi \mathrm{sr})(d / 5 \mathrm{kpc})^{2}$. This efficiency, as well as the measured power-law photon index (Table 1), are consistent with those observed for the magnetospheric components of other radio pulsars (Becker \& Trümper 1997). The detection of low duty-cycle X-ray pulsations could unambiguously confirm this interpretation. The current data cannot however rule out the possibility that the emission is coming from a very compact nebular region near the pulsar. At radio wavelengths, the head of the bow shock is only $\sim 1.5^{\prime \prime}$ from the pulsar (Gaensler \& Frail 2000). The reverse shock, which terminates the wind, is likely unresolvable in the Chandra image.

Marsden et al. (2001) suggested that PSR B1757-24 has a fallback disk from which it accretes, providing non-magnetic dipole spin-down torque to account for the possible difference between the pulsar's true and characteristic ages. They predict a thermal bremsstrahlung spectrum having $k T \sim$ $50 \mathrm{keV}$ and an X-ray luminosity (in an unspecified energy band) roughly an order of magnitude less than that predicted for magnetospheric emission. The Chandra detection we report here is consistent with standard magnetospheric emission. However, our data cannot rule out a fainter thermal component. 


\subsection{The Nebular Tail}

The tail X-ray emission is likely to be synchrotron radiation from the shocked pulsar wind. The pulsar wind appears to be confined by the ram pressure of the interstellar medium. If the pulsar's space velocity greatly exceeds the ambient medium's sound speed, a strong forward bow shock travels in front of it, and a reverse shock closer to the pulsar terminates its relativistic wind.

Conventional wisdom suggests that X-ray synchrotron nebulae should be smaller than the corresponding radio nebulae because radio electrons have much longer lives than do X-rays. This is consistent with the apparent contraction of the Crab nebula with increasing photon energy. However, for PSR B1757-24, Figures 1 and 2 demonstrate that the observed X-ray tail extends nearly $20^{\prime \prime}(0.48(d / 5 \mathrm{kpc}) \mathrm{pc})$ to the east of the pulsar, nearly as long as the detected radio tail (which, $\sim 10^{\prime \prime}$ further east, suddenly expands to form the flat-spectrum bubble, G5.27-0.90; Frail $\&$ Kulkarni 1991). The $5 \sigma$ upper limit on the proper motion implies a transverse velocity of $v_{t}<590 \mathrm{~km} \mathrm{~s}^{-1}$ (Gaensler \& Frail 2000). Thus, the time since the pulsar was at the eastern-most tip of the observed X-ray emission must be $>800 \mathrm{yr}$. The synchrotron lifetime of a photon of energy $E$ (in keV) in a magnetic field $B_{-4}$ (in units of $10^{-4} \mathrm{G}$ ) is $t_{s} \simeq 40 E^{-1 / 2} B_{-4}^{-3 / 2} \mathrm{yr}$. Thus, for $t_{s}>800 \mathrm{yr}$ and $E \simeq 1-9 \mathrm{keV}, B<0.8-14 \mu \mathrm{G}$. This is much less than the equipartition magnetic field $B_{\text {eq }} \sim \sqrt{\dot{E} / r_{s}^{2} c} \sim 70 \mu \mathrm{G}$ expected in the vicinity of the pulsar. Here, $r_{s}$ is the distance from the pulsar to the bow shock head, the approximate scale over which equipartition should hold (Kennel \& Coroniti 1984). This assumes the flow is subsonic, being confined on the trailing side by the high SNR pressure. This assumption seems reasonable, as in the absence of such confinement, it is hard to understand why this pulsar, which has space velocity not much larger than the average pulsar (e.g. Lyne \& Lorimer 1994), should have such a strking ram-pressure confined PWN. Hence, the X-ray tail behind PSR B1757-24 is unlikely to be a trail in the conventional sense, in that it cannot be synchrotron emission from pulsar wind particles just left behind after the passage of the pulsar.

Rather, assuming the spectrum of the tail is indeed non-thermal, freshly shocked wind particles must be continuously fed eastward with a velocity much larger than the pulsar space velocity, $v_{f} \gg v_{t}$. This is similar to the picture suggested for a putative X-ray tail behind PSR B1929+10 (see Wang et al. 1993, although that tail has not been confirmed) and a cometary-shaped PWN in the LMC SNR N157B (Wang \& Gotthelf 1998). However, Lyutikov (in preparation) has developed an alternative model of the structure of the tail region. In this model, the pulsar wind is shocked near the pulsar and forms a subsonically expanding tail confined by ISM ram pressure (without forming a de Laval nozzle as suggested by Wang et al. 1993). The typical velocities of the flow are weakly relativistic near the pulsar, decreasing downstream as the tail expands. At its end, the tail flow finally forms a pressure confined expanding bubble, presumably G5.27-0.90.

We can constrain the flow velocity $v_{f}$ of the wind particles in the tail by noting that it must be high enough to continuously supply particles given their cooling times. Thus, the flow time $t_{f} \lesssim t_{s}$. Assuming that the magnetic field reaches its equipartition value near the bow-shock 
head and that this value holds for the approximately one-dimensional tail region too, we find $t_{s} \gtrsim 70(E / 1 \mathrm{keV})^{-1 / 2}\left(B_{\text {eq }} / 70 \mu \mathrm{G}\right)^{-3 / 2} \mathrm{yr}$. As the tail extends to $0.48 \mathrm{pc}$ for $d=5 \mathrm{kpc}$, this implies $v_{f} \gtrsim 6800(d / 5 \mathrm{kpc})(E / 1 \mathrm{keV})^{1 / 2}\left(B_{\mathrm{eq}} / 70 \mu \mathrm{G}\right)^{3 / 2} \mathrm{~km} \mathrm{~s}^{-1}$.

The tail emission has low flux. The efficiency with which the pulsar's $\dot{E}$ is converted into tail X-rays in the $2-8 \mathrm{keV}$ band is only $0.00011(d / 5 \mathrm{kpc})^{2}$, roughly half of the point-source efficiency. This is in contrast to other rotation-powered pulsars, like the Crab, whose X-ray nebular emission is much brighter than the point-source output. Without timing information, we cannot rule out an ultra-compact nebula as the source of the point-source emission. But even so, the total efficiency for conversion of spin-down energy to nebula energy would be significantly less than that of most rotation-powered pulsars (Becker \& Trümper 1997).

Several effects may reduce the X-ray efficiency of ram pressure confined PWNs. First, the efficiency of conversion of $\dot{E}$ into X-ray emitting particles may be lower since the reverse shock in the ram-pressure-confined PWNs is strong only in the forward part of the head, subtending a much smaller solid angle than in a static PWN. Second, the low X-ray efficiency is expected if the flow time of the relativistic plasma through the tail is shorter than the synchrotron life time. In this case the particles will be able to emit only a small fraction of the energy that they acquired during acceleration at the reverse shock; most of the energy of the wind will be spent on doing work inflating the bubble at the end of the tail. Indeed, a similar argument was put forth Chevalier (2000) to explain the low efficiencies of the Vela and CTB 80 pulsars, both of which exhibit bow-shock morphologies and have relatively flat spectra. Finally, it is also possible that low surface brightness emission from beyond the eastern tip of the observable X-ray tail, or even from G5.27-0.90, have gone undetected in our observation. For example, emission from the direction of that nebula, having X-ray surface brightness half of the ACIS-S3 background, would contribute roughly two orders of magnitude more flux.

We can compare the observed X-ray tail flux with that predicted from the radio flux density and spectrum. Frail \& Kulkarni (1991) found that the radio tail has a flat spectrum $(\alpha \simeq 0$, where $\alpha$ is the energy spectral index), consistent with what we find at X-ray energies (§2). However, it is impossible to produce a continuous spectrum for the tail between the radio, where $f_{20 \mu \mathrm{eV}}=2.4 \times 10^{-8} \mathrm{erg} \mathrm{cm}^{-2} \mathrm{~s}^{-1} \mathrm{keV}^{-1}$ (Frail \& Kulkarni 1991), and X-ray, where $f_{5 \mathrm{keV}} \approx$ $1.6 \times 10^{-14} \mathrm{erg} \mathrm{cm}^{-2} \mathrm{~s}^{-1} \mathrm{keV}^{-1}(\S 2)$ bands. Thus there must be an intrinsic spectral break, which, even if it lies as low as in the radio band (e.g. $5 \mathrm{GHz}$ ), demands an X-ray photon index $>1.8$, much larger than expected for a synchrotron cooling break, and only marginally consistent with the data $(\S 2)$. Therefore, the broadband spectrum has at least two inflection points between the radio and X-ray bands, i.e. the emission in the two bands originates from separate populations of accelerated particles. The same problem arises in the Crab nebula (e.g. see Kennel \& Coroniti 1984). 


\section{The Supernova Remnant}

We have detected no X-ray emission from SNR G5.4-1.2. Assuming an association, if the pulsar's characteristic age is a good estimate of the true age, then the SNR is young $\left(t_{\mathrm{SNR}} \sim 16 \mathrm{kyr}\right)$, and we might expect thermal X-ray emission from hot gas behind the SNR shock. We considered a $4^{\prime} \times 4^{\prime}$ region of the SNR centered on coordinates (J2000) RA $18^{\mathrm{h}} 01^{\mathrm{m}} 18^{\mathrm{s}}$, DEC $-24^{\circ} 51^{\prime} 10^{\prime \prime}$. A background correction was applied using a region having the same dimensions next to the source region, scaling for the different effective areas. We find no excess emission in this region, with a $5 \sigma$ upper limit on the count rate of $0.017 \mathrm{cts} \mathrm{s}^{-1}$ in the range $0.3-8.0 \mathrm{keV}$. Assuming $d=5 \mathrm{kpc}$, the shock velocity in the Sedov solution should be $\approx 600 \mathrm{~km} \mathrm{~s}^{-1}$, corresponding to a shock temperature $k T \approx 0.5 \mathrm{keV}$. Using a Raymond-Smith model with $k T=0.5 \mathrm{keV}$ and $N_{H}=3 \times 10^{22} \mathrm{~cm}^{-2}$, and assuming the area under consideration encloses a volume $\sim 6 \times 10^{57} \mathrm{~cm}^{3}$, the limit on the count rate in this region corresponds to $n_{e} n_{H}<0.15 \mathrm{~cm}^{-6}$, where $n_{e}$ and $n_{H}$ are the electron and hydrogen densities, respectively. Assuming a composition of pure ionized hydrogen, the upper limit on the pre-shock density is $n_{H}<0.1 \mathrm{~cm}^{-3}$. This result is not a strong function of the assumed temperature: for $k T=0.2(5) \mathrm{keV}$, we find $n_{H}<0.50(0.04) \mathrm{cm}^{-3}$. These limits are unconstraining. If the system has an age of $16 \mathrm{kyr}$, the inferred density is $n_{H} \sim 0.003 \mathrm{~cm}^{-3}$ (Frail \& Kulkarni 1991). If the SNR is much older than indicated by the pulsar's spin-down (Gaensler \& Frail 2000), then the SNR

expansion speed is $<100 \mathrm{~km} \mathrm{~s}^{-1}$, and any X-ray emission from the SNR is expected to be too faint to detect for this distance and absorption.

We thank M. Roberts for useful discussions. This work is supported by SAO grant GO0-1133A, and by NASA LTSA grant NAG5-8063 and NSERC grant Rgpin 228738-00 to V.M.K. E.V.G is supported by the NASA LTSA grant NAG5-22250. B.M.G. acknowledges the support of a Hubble Fellowship awarded by STScI.

\section{REFERENCES}

Becker, W. \& Trümper, J. 1997, A\&A, 326, 682

Burke, B. E., Gregory, J., Bautz, M. W., Prigozhin, G. Y., Kissel, S. E., Kosicki, B. N., Loomis, A. H., \& Young, D. J. 1997, IEEE Transac. Elec. Devices, 44, 1633

Caswell, J. L., Kesteven, M. J., Komesaroff, M. M., Haynes, R. F., Milne, D. K., Stewart, R. T., \& Wilson, S. G. 1987, MNRAS, 225, 329

Chevalier, R. A. 2000, ApJ, 539, L45

Cordes, J. M., Romani, R. W., \& Lundgren, S. C. 1993, Nature, 362, 133

Deutsch, E. W. 1999, Astron. J., 118, 1882

Frail, D. A., Kassim, N. E., \& Weiler, K. W. 1994, Astron. J., 107, 1120 
Frail, D. A. \& Kulkarni, S. R. 1991, Nature, 352, 785

Gaensler, B. M. \& Frail, D. A. 2000, Nature, 406, 158

Kennel, C. F. \& Coroniti, F. V. 1984, ApJ, 283, 710

Lyne, A. G. \& Lorimer, D. R. 1994, Nature, 369, 137

Manchester, R. N., D’Amico, N., \& Tuohy, I. R. 1985, MNRAS, 212, 975

Manchester, R. N., Kaspi, V. M., Johnston, S., Lyne, A. G., \& D’Amico, N. 1991, MNRAS, 253, $7 \mathrm{P}$

Marsden, D., Lingenfelter, R. E., \& Rothschild, R. E. 2001, ApJ, 547, L45

Monet, D., Bird, A., Canzian, B., Harris, H., Reid, N., Rhodes, A., Sell, S., Ables, H., Dahn, C., Guetter, H., Henden, A., Leggett, S., Levison, H., Luginbuhl, C., Martini, J., Monet, A., Pier, J., Riepe, B., Stone, R., Vrba, F., \& Walker, R. 1996, USNO-SA2.0, U.S. Naval Observatory, Washington DC

Wang, Q. D. \& Gotthelf, E. V. 1998, ApJ, 494, 623

Wang, Q. D., Li, Z.-Y., \& Begelman, M. C. 1993, Nature, 364, 127 
Table 1. Spectral Parameters for PSR B1757-24 for Two Trial Models.

\begin{tabular}{lcc}
\hline \hline & Power Law & Thermal Bremsstrahlung \\
\hline$N_{H}\left(10^{22} \mathrm{~cm}^{-2}\right)$ & $3.5_{-1.1}^{+1.3}$ & $3.3_{-1.0}^{+0.76}$ \\
$\Gamma^{\mathrm{b}}$ & $1.6_{-0.5}^{+0.6}$ & $\ldots$ \\
$k T(\mathrm{keV})$ & $\ldots$ & $16_{-8}^{+\infty}$ \\
Absorbed Flux & $5.4_{-0.5}^{+0.4}$ & $5.1_{-0.5}^{+0.4}$ \\
Unabsorbed Flux $^{\mathrm{c}}$ & $6.9_{-0.6}^{+0.5}$ & $6.6_{-0.6}^{+0.5}$ \\
$\chi_{\nu}^{2}$ (for 24 DoF) & 1.24 & 1.26 \\
\hline
\end{tabular}

${ }^{a}$ Uncertainties reported are at the $90 \%$ confidence level.

${ }^{\mathrm{b}}$ Photon index.

${ }^{\mathrm{c}}$ In $10^{-13} \mathrm{erg}^{-2} \mathrm{~s}^{-1}$ in the $2-10 \mathrm{keV}$ band. 


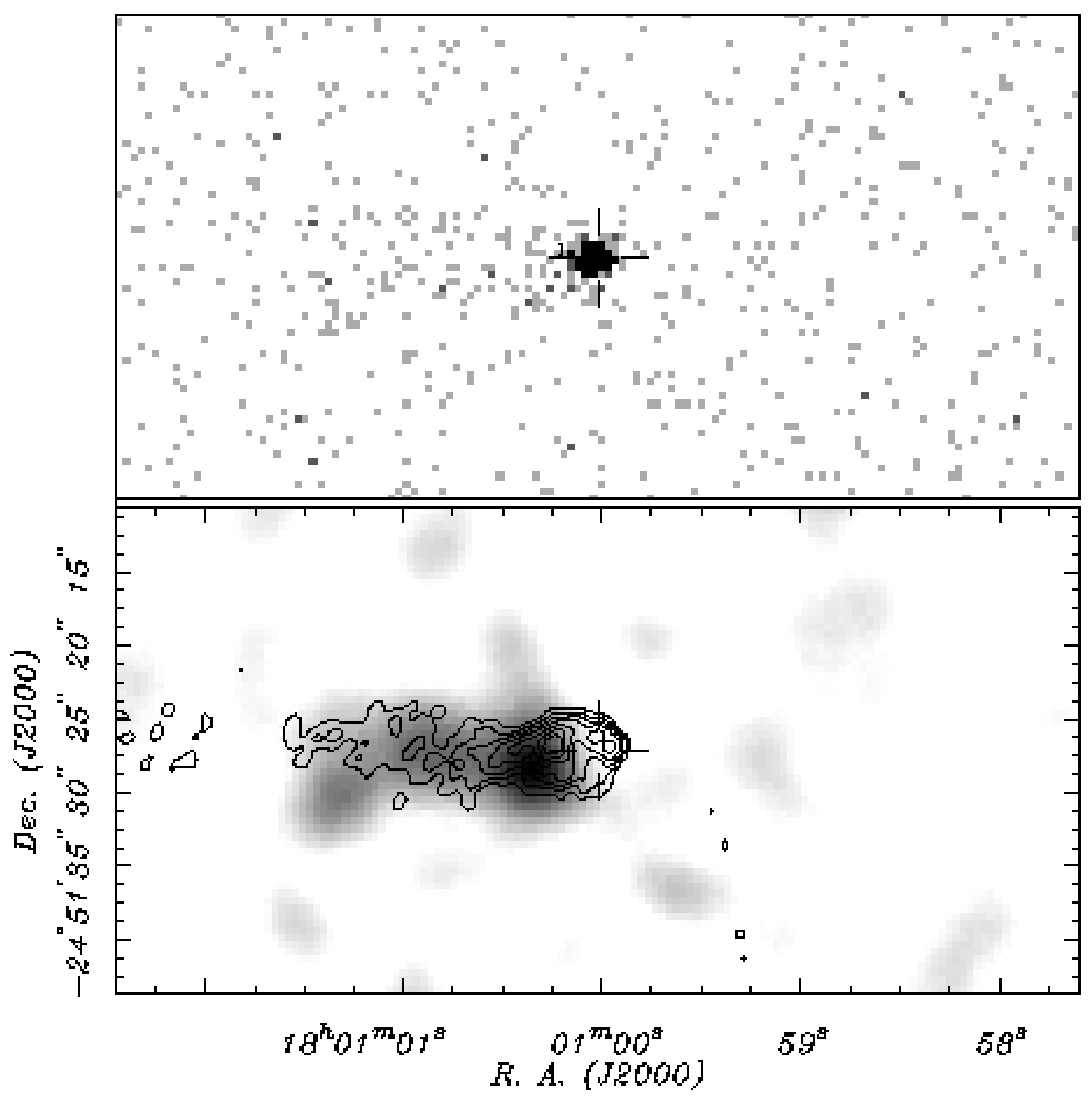

Fig. 1.- Chandra X-ray detection of PSR B1757-24 and its nebular tail. Top panel: greyscale plot of the $0.3-10 \mathrm{keV}$ image, centered on the pulsar, and scaled to show all pixels with detected photons. The X-ray point source is coincident with the radio pulsar position, denoted by the cross. The contours show $4.9 \mathrm{GHz}$ emission in equally spaced contours ranging from 0.17 to $0.56 \mathrm{mJy} / \mathrm{beam}$. The radio data have beam size $1^{\prime \prime} .2$ and were obtained at the VLA on 1999 October 23 by one of us (B. Gaensler). Bottom panel: same image with the X-ray point source contribution removed, the image smoothed with a Gaussian of width $\sim 2^{\prime \prime}$, and the intensity scaled to emphasize the diffuse $\mathrm{X}$-ray emission. 


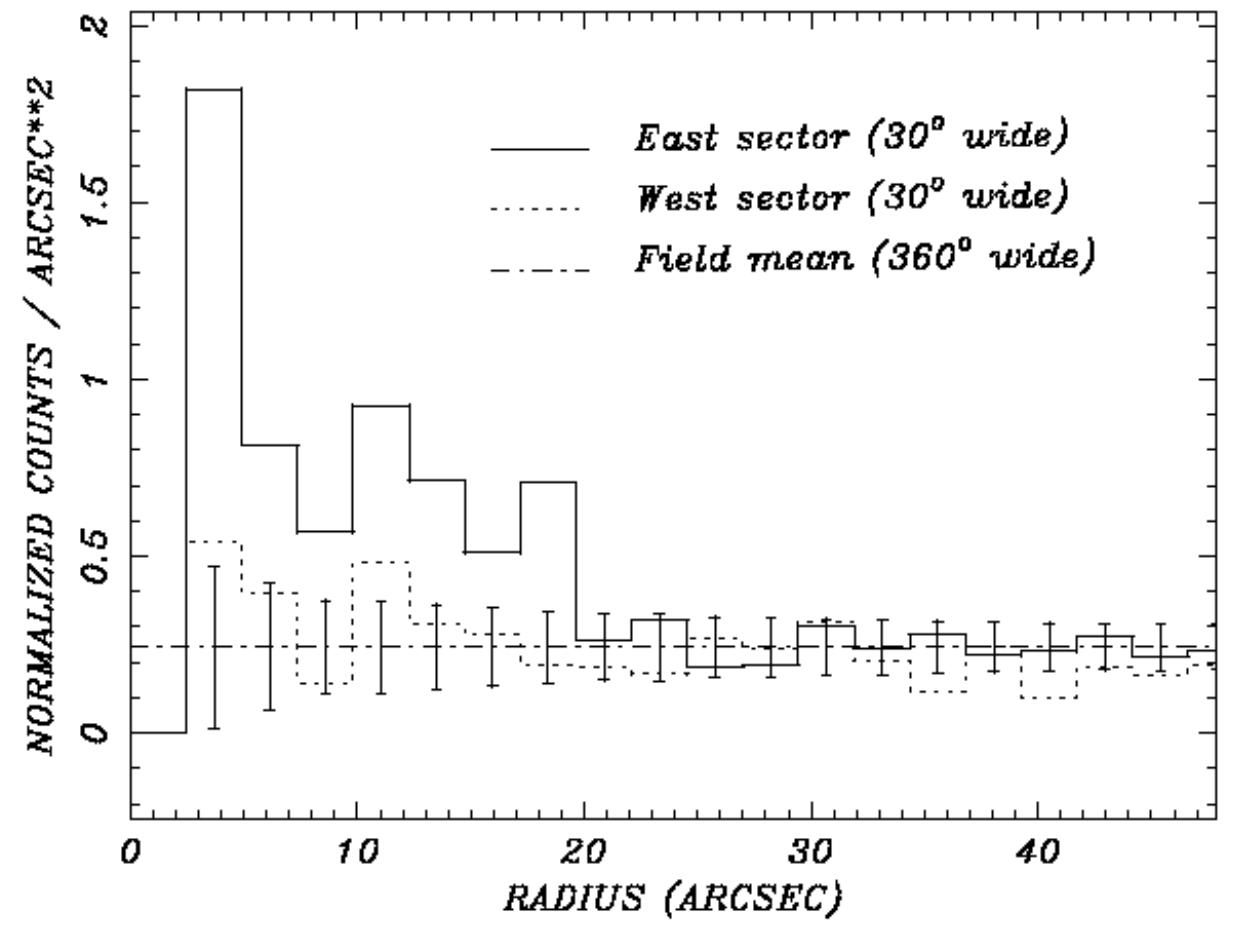

Fig. 2.- X-ray intensity as a function of radius from the point source in two $30^{\circ}$ wedges, one east (solid line) and one west (dotted line) of the pulsar. The point-source emission has been subtracted from the plot. The field mean is shown by a dot-dashed line. $1 \sigma$ error bars for each radial bin are shown. 